\title{
Enhanced paraconductivity-like fluctuations in the radiofrequency spectra of ultracold Fermi atoms
}

\author{
Pierbiagio Pieri ${ }^{\star}$, Andrea Perali and Giancarlo Calvanese Strinati
}

In gases of ultracold Fermi atoms, the crossover from Bardeen-Cooper-Schrieffer superconductivity to BoseEinstein condensation ${ }^{1-3}$ can be realized by continuously varying the attraction between fermions of different species ${ }^{4}$. In this context, radiofrequency spectroscopy ${ }^{5-7}$ provides a microscopic probe to infer the nature of fermionic pairing. In the regime of strong interaction, the pairing affects a wide temperature range, which includes the critical temperature $T_{c r}$ in analogy to the pseudogap physics for high-temperature superconductors. Here, we establish a direct connection between the theory of radiofrequency spectra for ultracold fermions above $T_{c}$ and the theory of paraconductivity fluctuations in superconductors. Our calculations compare favourably to available experimental radiofrequency spectra, and demonstrate that the role of fluctuations for ultracold fermions is considerably enhanced with respect to superconductors. In addition, we illustrate how to extract from the spectra an energy scale associated with pairing and relate it to a universal quantity recently introduced for Fermi gases ${ }^{8}$.

It is widely appreciated at present that ultracold Fermi atoms represent ideal systems for deepening our understanding of condensed-matter systems, especially as far as many-body quantum physics is concerned. This is because the inter-particle interaction and species populations can be fine-controlled and tuned almost at will, in such a way that the system Hamiltonian is precisely known over a wide parameter range. As experimental techniques are able to provide diverse and accurate data, major theoretical efforts can be justified to attempt detailed comparison with experiments, particularly for those aspects that have remained unsettled in condensed matter.

In this respect, radiofrequency experiments ${ }^{5-7}$ (especially in their tomographic version) provide fertile ground for exploring complementary aspects related to excitation gaps and pairing fluctuations, both below and above $T_{\mathrm{c}}$ for the transition to the superfluid phase in these neutral systems. A crucial aspect here is the occurrence of several hyperfine levels, which are split by a magnetic field and exhibit mutual Fano-Feshbach resonances that strongly amplify the two-body interaction when the field is varied ${ }^{4}$. The hyperfine levels act as components of a (hyper) spin and a spin-flip is generated by the radiofrequency transition, so that a dynamical (two-particle) spin-correlation function is effectively measured by radiofrequency spectra ${ }^{9}$.

Below $T_{c}$, theoretical interpretation of these spectra has revealed a competition between pairing-gap effects in the initial state of the transition and final-state effects, which tend to push the oscillator strength towards opposite sides of the spectrum ${ }^{10}$. In this case, the presence of a well-developed pairing gap at low temperature makes a Bardeen-Cooper-Schrieffer random-phase approximation (BCS-RPA) approach for response functions sufficiently accurate to reproduce the experimental features ${ }^{10}$.
When the temperature increases across $T_{\mathrm{c}}$, the pairing gap is replaced by pairing fluctuations, and precursor effects of pairing appear in the normal phase above $T_{\mathrm{c}}$. These effects manifest themselves at the simplest level in the single-particle spectral function with a depression of the spectral weight (pseudogap) at low frequencies for small enough wave vectors (the effect being amplified when approaching the Bose-Einstein condensation (BEC) limit of the BCS-BEC crossover ${ }^{11}$ ). In high-temperature cuprate superconductors (where pairing is competing with other kinds of ordering), photoemission (angle-resolved photoemission spectroscopy) experiments have extensively been used to unravel the nature of the pseudogap in single-particle excitations ${ }^{12}$. Recently, attempts to disentangle single-particle excitations from radiofrequency (two-particle) spectra have also been made for ultracold Fermi gases ${ }^{13}$, by resolving the wave vector of the photo-excited fermions.

Pairing fluctuations in the normal phase above $T_{\mathrm{c}}$ are known to affect the two-particle response of superconductors. In particular, for superconducting thin films the possibility of fluctuational creation of Cooper pairs has been shown to enhance their conductivity, an effect known as 'paraconductivity' from the work of Aslamazov and Larkin ${ }^{14}(\mathrm{AL})$. Another source of interaction of fermions with fluctuating Cooper pairs stems from the extra contribution to the conductivity found by Maki ${ }^{15}$ and Thompson ${ }^{16}$ (MT). The AL and MT contributions have been detected experimentally to produce small changes to the normal conductivity, which can be amplified by reducing the dimensionality ${ }^{17}$. Whereas the AL contribution to the conductivity can be obtained theoretically by a time-dependent generalization of the Ginzburg-Landau equations ${ }^{18}$, accounting for the MT contribution requires one to introduce finite-temperature Feynman diagrams for the current response function, a technique that yields also a third relevant contribution known as density-of-states (DOS) renormalization ${ }^{19}$.

Here, we calculate the corresponding fluctuation contributions to the dynamical spin-correlation function associated with radiofrequency spectra, by using finite-temperature Feynman diagrams in the normal phase. Our calculation reveals that, even in three dimensions, the role of pairing fluctuations can be drastically enhanced for the radiofrequency spin-correlation function of ultracold Fermi atoms with respect to the current-correlation function of superconductors. The results of our calculation compare favourably to the available radiofrequency experimental data for Fermi gases with balanced populations under various coupling conditions.

The differences between the current- and radiofrequency spincorrelation functions originate from the different nature of the coupling to the external probe, the involvement of a third spin component and the wider coupling range that can be explored by means of Fano-Feshbach resonances with Fermi atoms. Apart from these differences (which yet make the MT contribution vanish 


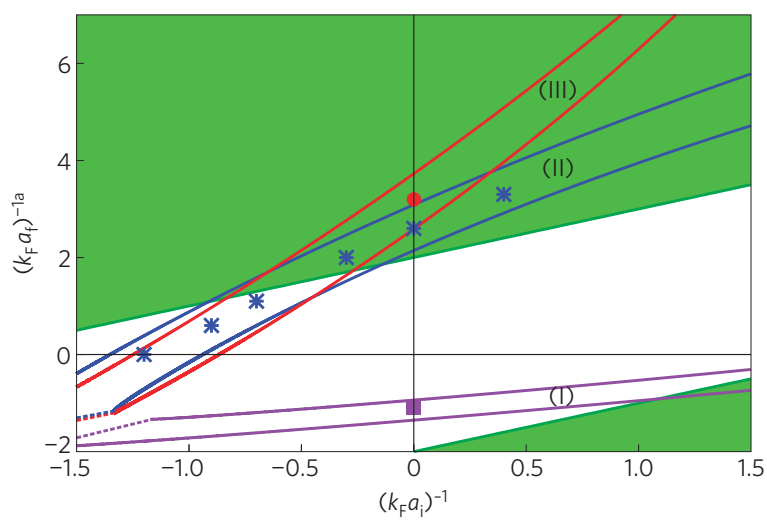

Figure 1 | Allowed coupling values for ${ }^{6} \mathrm{Li}$ in the $\left(\left(k_{\mathrm{F}} a_{\mathrm{i}}\right)^{-1},\left(k_{\mathrm{F}} a_{\mathrm{f}}\right)^{-1}\right)$ plane. Radiofrequency experiments realized with ultracold ${ }^{6} \mathrm{Li}$ atoms correspond to the three following combinations of scattering lengths: (I) for $\left(a_{i}=a_{12}, a_{f}=a_{13}\right)$, (II) for $\left(a_{i}=a_{13}, a_{f}=a_{12}\right)$ and (III) for $\left(a_{i}=a_{13}, a_{f}=a_{23}\right)$. Using the values of these scattering lengths versus the magnetic field given in ref. 29 and the experimentally accessible values of the Fermi wave vector $k_{\mathrm{F}}=\left(3 \pi^{2} n\right)^{1 / 3}$, where $n$ is the (total) density at the trap centre (typically, $k_{\mathrm{F}} \approx 2.7 \times 10^{-4} a_{0}{ }^{-1}$, where $a_{0}$ is the Bohr radius), we construct the three stripes labelled (I), (II) and (III) inside which radiofrequency spectra can be collected, the widths of which correspond to an estimated $40 \%$ variation on the values of $k_{F}$. The symbols correspond to the three different sets of tomographic radiofrequency spectra reported in ref. 7. The green area indicates the region $2 \lesssim\left|\left(k_{\mathrm{F}} a_{\mathrm{i}}\right)^{-1}-\left(k_{\mathrm{F}} a_{\mathrm{f}}\right)^{-1}\right|$ where it is possible to extract the quantity $\Delta_{\infty}$ (see the main text) from the high-frequency tail of the radiofrequency spectra.

identically for the radiofrequency spin-correlation function), the topological structure of the Feynman diagrams for the AL and DOS contributions remains the same for both calculations (see the Methods section).

For the radiofrequency spectra of interest here, two spin components $(\alpha$ and $\beta$ ) are initially equally populated whereas a third $(\gamma)$ is empty. For a transition that flips $\beta$ into $\gamma$, the relevant scattering lengths are then $a_{\alpha \beta}$ for the initial (i) and $a_{\alpha \gamma}$ for the final (f) state. In our calculation, the DOS contribution contains only pairing fluctuations related to $a_{\alpha \beta}$ (initial-state effects), whereas the AL contribution contains in addition pairing fluctuations related to $a_{\alpha \gamma}$ (final-state effects) — see the Methods section. For this reason, we refer to $a_{\alpha \beta}$ as $a_{\mathrm{i}}$ and to $a_{\alpha \gamma}$ as $a_{\mathrm{f}}$. Experiments have been able to explore different combinations of these spin components, thereby enlarging the accessible coupling ranges. Figure 1 reports the corresponding locations of the possible experimental coupling values for ${ }^{6} \mathrm{Li}$ in the $\left(\left(k_{\mathrm{F}} a_{\mathrm{i}}\right)^{-1},\left(k_{\mathrm{F}} a_{\mathrm{f}}\right)^{-1}\right)$ plane, where $k_{\mathrm{F}}$ is the Fermi wave vector.

Comparisons between the results of our numerical calculations and the experimental spectra for ${ }^{6} \mathrm{Li}$ are reported in Fig. 2, resulting in quite good agreement between theory and experiments. Recall that, in the absence of pairing fluctuations, a single delta-spike at zero frequency would absorb the whole spectra weight (the value of which is dictated by a sum rule for the spectral area ${ }^{10}$ and is not changed by further inclusion of $a_{\mathrm{i}}$ and $a_{\mathrm{f}}$ ). In all panels, the left peak is associated with the presence of a localized (bound) final state, which typically absorbs a spectral weight comparable to the right peak. The latter originates from continuum transitions and comprises the high-frequency tail. For these reasons, the final-state effects associated with the AL contribution have an essential role in all cases (see also Supplementary Fig. S1). Nonetheless, when the bound and continuum peaks are sufficiently apart, the continuum part of the spectra seems to be only slightly modified by the inclusion of final-state effects provided the peak heights (obtained by DOS alone and by DOS plus AL) are brought to coincide.

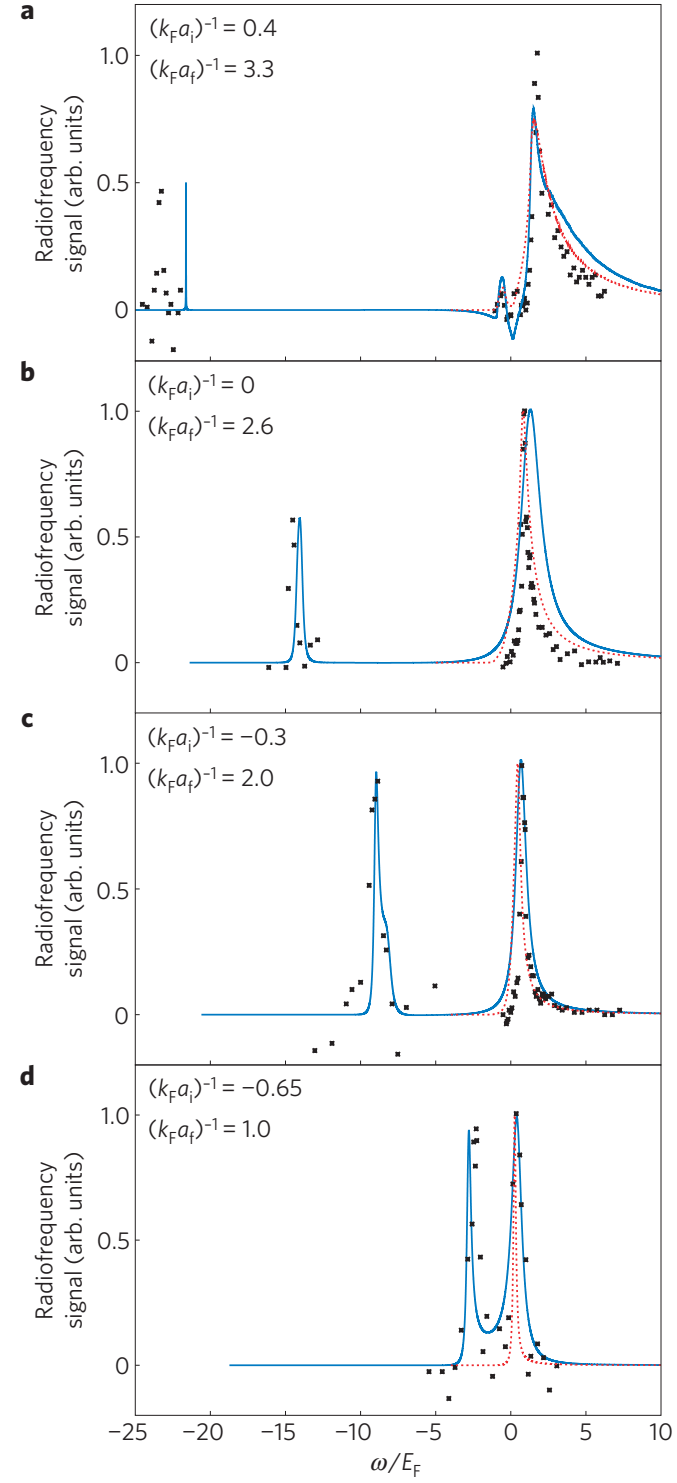

Figure 2 | Comparison between theoretical and experimental radiofrequency spectra. a-d, The experimental spectra from Figs. 4a-d of ref. 7 (black squares) compared to our calculations, with the inclusion of pairing fluctuations due to $a_{\mathrm{i}}$ (red dotted curves: DOS contribution) and with the extra inclusion of pairing fluctuations due to $a_{f}$ (blue solid curves: DOS plus AL contributions). The experimental spectra (tomographic for a-c, and trap-averaged for $\mathbf{d}$ ) span approximately the temperature range $\left(0.7 T_{c}-T_{c}\right)$, and reveal a smooth evolution across $T_{c}$. The theoretical spectra are calculated for a homogeneous system in the normal phase at a temperature $T\left(=1.1 T_{\mathrm{c}}\right)$ slightly above $T_{\mathrm{c}}$. In a-c, the bound (left) and continuum (right) peaks of the experimental spectra were independently normalized to the corresponding peak heights, whereas in $\mathbf{d}$ a single normalization was used ${ }^{7}$. We have adopted the same procedure for the comparison. Our calculation provides for the relative weight of the bound peak the values $59 \%(\mathbf{a}), 44 \%$ (b) and $33 \%$ (c). In a, the discrepancy between the positions of the experimental and theoretical bound peaks can be accounted for by a molecular multi-channel calculation ${ }^{30}$. The couplings in a-c correspond to those of Figs. $4 a-c$ of ref. 7, whereas the coupling in $\mathbf{d}$ is obtained from the value of $k_{F}$ at the trap centre, estimated from the trap-averaged value $k_{\mathrm{F}}^{\prime}$ given in Fig. $4 \mathrm{~d}$ of ref. 7.

These considerations justify a posteriori the procedure adopted in ref. 7 to fit the continuum part of the experimental spectra disregarding final-state effects. 

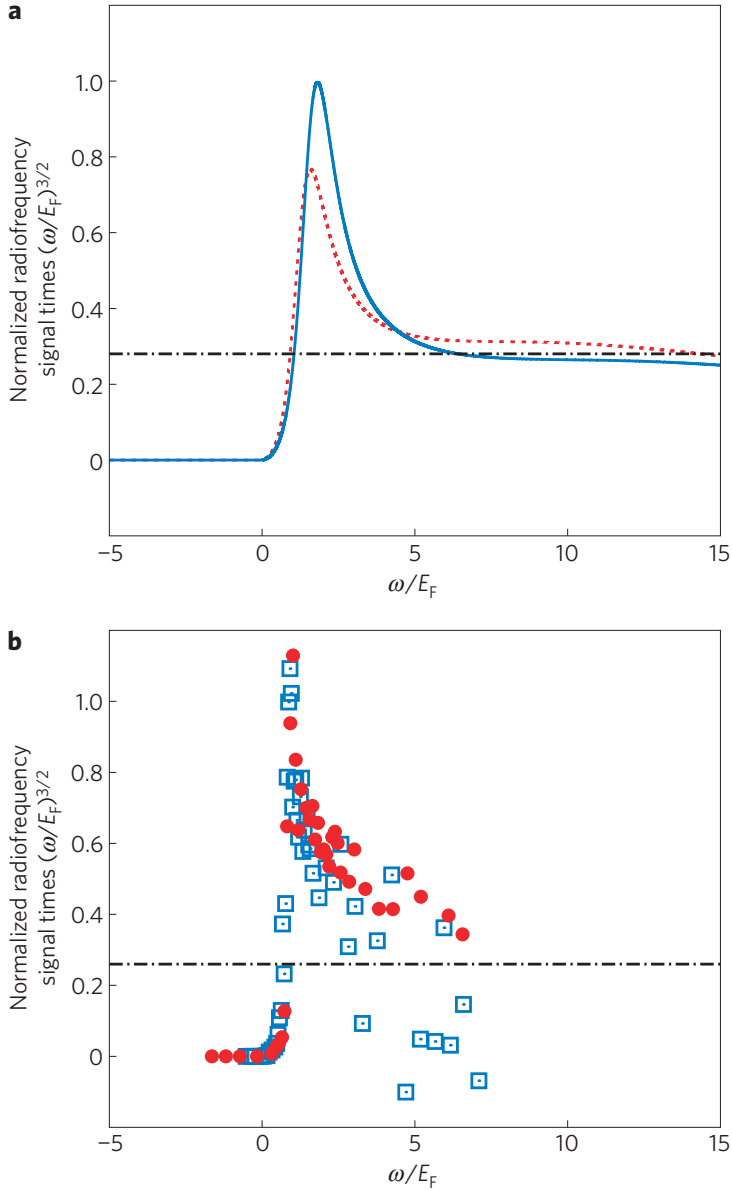

Figure 3 | Procedure for extracting $\Delta_{\infty}$ from the tail of the

radiofrequency spectra. $\mathbf{a}$, Theoretical radiofrequency spectra normalized to the weight $A$ of the continuum peak and multiplied by $\left(\omega / E_{\mathrm{F}}\right)^{3 / 2}$. The blue solid curve is for $\left(k_{F} a_{f}\right)^{-1}=2.6$ (with $A=56 \%$ ) and the red dotted curve is for $\left(k_{\mathrm{F}} a_{\mathrm{f}}\right)^{-1}=3.2$ (with $A=63 \%$ ), and $\left(k_{\mathrm{F}} a_{\mathrm{i}}\right)^{-1}=0$ and $T=1.1 T_{\mathrm{c}}$ in both cases. The horizontal line corresponds to the value $\Delta_{\infty}=0.73 \mathrm{EF}_{\mathrm{F}}$ obtained at the same temperature. $\mathbf{b}$, An analogous procedure is applied to the corresponding experimental spectra, by normalizing the area of the continuum peak to unity. The blue open squares are from Fig. $2 b$ of ref. 7 with $\left(k_{F} a_{f}\right)^{-1}=2.6$ and the red filled circles are from Supplementary Fig. S1 of ref. 7 with $\left(k_{\mathrm{F}} a_{\mathrm{f}}\right)^{-1}=3.2$, and $\left(k_{\mathrm{F}} a_{\mathrm{i}}\right)^{-1}=0$ and the temperature is below (but close to) $T_{\mathrm{c}}$ in both cases. The data yield approximately the value $0.25 \pm 0.10$ for the height of the plateau, from which we get $\Delta_{\infty} / E_{\mathrm{F}}=0.69_{-0.16}^{+0.12}$ with no input from theory.

In Fig. 2, the experimental spectra taken slightly below $T_{c}$ have been compared with our calculations done slightly above $T_{\mathrm{c}}$. This comparison is meaningful because the DOS and AL contributions considered here contain non-critical fluctuations with short spatial range, and thus smoothly evolve across $T_{\mathrm{c}}$. When lowering the temperature further, however, the BCS-RPA response from the BCS mean field becomes important, as was shown in ref. 10 where account was given of experimental data for $T \lesssim 0.5 T_{\mathrm{c}}$. The BCS-RPA approach applies, in particular, to $T=0$, where it reduces to that of ref. 20. The pairing fluctuations contained in the DOS plus AL contributions thus replace at high-enough temperature those contained in the BCS-RPA response at low temperature. For this reason, the BCS-RPA response when applied to temperatures above $T_{\mathrm{c}}$ (see ref. 21) fails to even qualitatively account for the radiofrequency spectra (see Supplementary Fig. S2).

Experimentally, the motivation for exploring radiofrequency spectroscopy with ultracold Fermi atoms ${ }^{5}$ stemmed from the

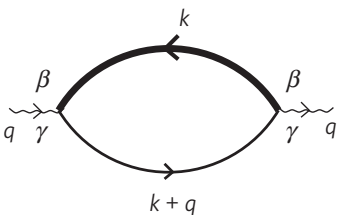

b
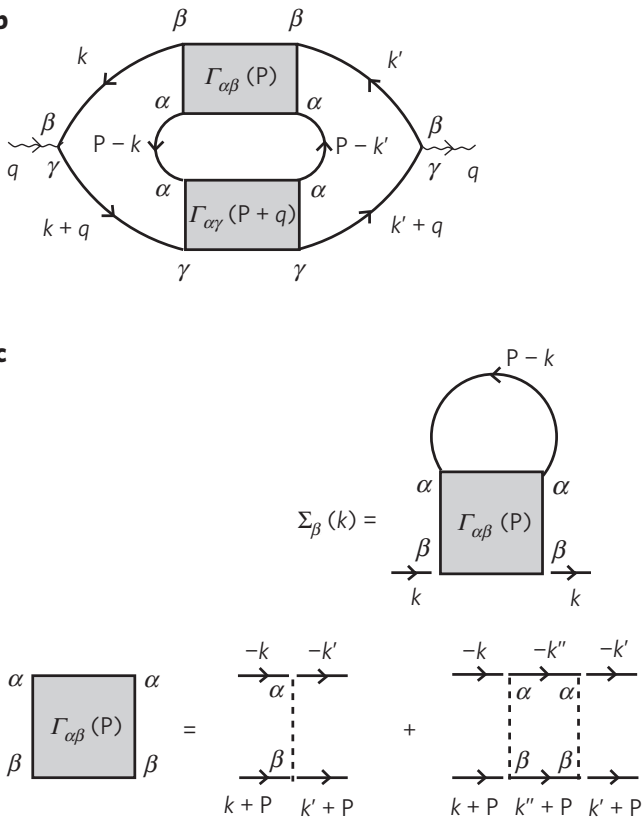

Figure 4 | Diagrammatic representation of the spin-correlation function above $\boldsymbol{T}_{\mathbf{c}}$. $\mathbf{a}$, DOS contribution where the fermionic $\beta$ - $\beta$ single-particle propagator (upper line) is dressed by the self-energy $\Sigma_{\beta}$, which includes pairing fluctuations of the initial state $\beta$ with its mate $\alpha$ through the pairing propagator $\Gamma_{\alpha \beta} . \mathbf{b}, \mathrm{AL}$ contribution that includes, in addition, pairing fluctuations of the final state $\gamma$ with the mate $\alpha$ left behind. $\mathbf{c}$, Self-energy $\Sigma_{\beta}$ and pairing propagator $\Gamma_{\alpha \beta}$ between spins $\alpha$ and $\beta$ in the initial state ( $\Gamma_{\alpha \gamma}$ between spins $\alpha$ and $\gamma$ in the final state is similarly obtained). The solid and dashed lines stand for fermionic single-particle propagators and interactions, respectively, and the labels attached to the end points identify the relevant spins. All diagrams are drawn in four-momentum space. Only the single-particle propagator $\beta-\beta$ in $\mathbf{a}$ is dressed by self-energy insertions.

expectation of extracting the value of a pairing gap directly from the (continuum part of the) spectra. This would be possible within a BCS description at low temperature, because in this case the continuum edge occurs at $\Delta^{2} /\left(2 E_{\mathrm{F}}\right)$, where $\Delta$ is the BCS gap parameter and $E_{\mathrm{F}}=k_{\mathrm{F}}^{2} /(2 m)$ is the Fermi energy ( $m$ being the fermion mass). Such a simplified picture, however, does not apply to the unitarity regime about $\left(k_{\mathrm{F}} a_{\mathrm{i}}\right)^{-1}=0$ owing to strong-coupling effects in the initial and/or final states of the transition. Accordingly, no procedure has been suggested so far for extracting from the radiofrequency spectra an energy scale associated with pairing interaction in systems with balanced populations ${ }^{22}$.

The present analysis allows us to identify such an energy scale, when the bound and continuum peaks of a radiofrequency spectrum are sufficiently far apart as in the experiments of ref. 7 or, more generally, when $2 \lesssim\left|\left(k_{\mathrm{F}} a_{\mathrm{i}}\right)^{-1}-\left(k_{\mathrm{F}} a_{\mathrm{f}}\right)^{-1}\right|$, corresponding to the green area in Fig. 1. In this case, there exists a window on the high-frequency side of the continuum peak where the radiofrequency signal behaves according to $B\left(\omega / E_{\mathrm{F}}\right)^{-3 / 2}$ with $B=A\left(3 / 2^{5 / 2}\right)\left(\Delta_{\infty} / E_{\mathrm{F}}\right)^{2}$. Here, $A$ stands for the area of the continuum peak once the total area of the radiofrequency spectrum is taken to be unity. Typically, this frequency window extends between a few times $E_{\mathrm{F}}$ and $\left(m a_{\mathrm{f}}^{2}\right)^{-1}$, past which the spectral tail 
is dominated by final-state effects and decays as $\omega^{-5 / 2}$ (such that, if final-state effects were totally neglected $\left(a_{\mathrm{f}}=0\right)$ the $\omega^{-3 / 2}$ tail would extend up to infinity). The above expression is written in analogy with the high-frequency behaviour of the radiofrequency signal within the BCS approximation, in which $\Delta$ appears in place of $\Delta_{\infty}$. Physically, although $\Delta$ is a mean-field quantity, the energy scale $\Delta_{\infty}$ results from adding pairing fluctuations beyond the mean field and thus remains meaningful even above the critical temperature. The energy scale $\Delta_{\infty}$ can be further related to the asymptotic behaviour $C / \mathbf{k}^{4}$ of the wave-vector distribution function $n(\mathbf{k})$, where $C$ is the 'contact intensity' introduced in ref. 8, which enters several quantities of a Fermi gas in a universal way ${ }^{23,24}$. On general grounds, we identify $C=\left(m \Delta_{\infty}\right)^{2}$ (see the Methods section).

In Fig. 3a, the theoretical radiofrequency spectra (once multiplied by $\left.\left(\omega / E_{\mathrm{F}}\right)^{3 / 2}\right)$ identify a plateau beginning at $\omega \simeq 5 E_{\mathrm{F}}$, the height of which is $\left(3 / 2^{5 / 2}\right)\left(\Delta_{\infty} / E_{\mathrm{F}}\right)^{2}$ according to the above argument. This height is compared with the horizontal line, obtained by the value of $\Delta_{\infty} / E_{\mathrm{F}}$ resulting from our independent calculation of the contact intensity $C$ through $n(\mathbf{k})$. In Fig. 3b, we attempt the same procedure for the experimental radiofrequency spectra. Even though the signal-to-noise ratio seems too low to draw definite conclusions, a numerical estimate for $\Delta_{\infty} / E_{\mathrm{F}}$ can still be extracted within some uncertainty related to the determination of the value of the plateau level (the presence of which is expected on theoretical grounds). Future experiments with improved statistics on the wings of the radiofrequency spectra could unambiguously identify the value of this plateau, thus mapping out the value of $\Delta_{\infty}$ (and therefore the contact intensity $C$ of ref. 8 ) in extended temperature and coupling ranges.

The results obtained here demonstrate the important role of pairing fluctuations for strongly interacting Fermi systems, which smoothly evolve across $T_{\mathrm{c}}$ owing to their short spatial range. In this respect, our results emphasize how local (pairing) fluctuations get amplified by the strong interaction, irrespective of the presence of critical fluctuations. This is also reflected in the wide temperature range well above the critical temperature where the response of the Fermi system is influenced by fluctuations. At the same time, our calculations, being motivated by the need to get a valuable comparison between theory and experiments in systems such as ultracold fermions for which the Hamiltonian is known, have contributed to advance the current state-of-the-art of quantum many-body physics.

\section{Methods}

Radiofrequency spectrum and the spin-correlation function. The radiofrequency spectrum is obtained theoretically within linear-response theory as:

$$
\delta\left\langle I\left(\omega_{\mathrm{th}}\right)\right\rangle=-2 g^{2} \int \mathrm{d} \mathbf{r d} \mathbf{r}^{\prime} \operatorname{Im}\left\{\Pi_{\beta \gamma}^{\mathrm{R}}\left(\mathbf{r}, \mathbf{r}^{\prime} ; \omega_{\mathrm{th}}\right)\right\}
$$

where $g$ is the coupling constant of the atomic $(\beta \rightarrow \gamma)$ transition and $\Pi_{\beta \gamma}^{\mathrm{R}}\left(\mathbf{r}, \mathbf{r}^{\prime} ; \omega_{\text {th }}\right)$ is the Fourier transform of the retarded correlation function $\prod_{\beta \gamma}^{\mathrm{R}}\left(\mathbf{r}, \mathbf{r}^{\prime} ; t-t^{\prime}\right)=-i \theta\left(t-t^{\prime}\right)\left\langle\left[B_{\beta \gamma}(\mathbf{r}, t), B_{\beta \gamma}^{\dagger}\left(\mathbf{r}^{\prime}, t^{\prime}\right)\right]\right\rangle$ taken at the shifted frequency $\omega_{\mathrm{th}}=\omega_{\mathrm{RF}}+\mu_{\beta}-\mu_{\gamma}$ with respect to the frequency of the radiofrequency fields, $\omega_{\mathrm{RF}}$ (we set $\hbar=1$ throughout). Here, $B_{\beta \gamma}^{\dagger}(\mathbf{r})=\psi_{\gamma}^{\dagger}(\mathbf{r}) \psi_{\beta}(\mathbf{r})$ is the transition operator in terms of the field operators $\psi_{\beta / \gamma}$ at the spatial point $\mathbf{r}, \mu_{\beta}$ and $\mu_{\gamma}$ are the chemical potentials for the initial (equally populated) and final (empty) levels, in the order, and $\langle\cdots\rangle$ stands for a thermal average.

Following a standard procedure, the retarded correlation function is conveniently calculated by means of its Matsubara counterpart

$$
\Pi_{\beta \gamma}\left(\mathbf{r}, \mathbf{r}^{\prime} ; \Omega_{\nu}\right)=\int_{0}^{\beta} \mathrm{d} \tau \mathrm{e}^{i \Omega_{\nu} \tau}\left\langle\mathrm{T}_{\tau}\left[\psi_{\beta}\left(\mathbf{r}^{\prime}, 0\right) \psi_{\beta}^{\dagger}\left(\mathbf{r}, \tau^{+}\right) \psi_{\gamma}(\mathbf{r}, \tau) \psi_{\gamma}^{\dagger}\left(\mathbf{r}^{\prime}, 0^{+}\right)\right]\right\rangle
$$

as this expression admits a representation in terms of Feynman diagrams. Here, $\mathrm{T}_{\tau}$ is the time-ordering operator for imaginary time $\tau$ and $\Omega_{\nu}=2 \pi \nu T$ ( $v$ integer) is a bosonic Matsubara frequency at temperature $T$ (we set $k_{B}=1$ throughout). Above $T_{\mathrm{c}}$, the relevant Feynman diagrams for the two-particle response are the DOS and AL contributions shown in Fig. 4, which result once the single-particle self-energy with pairing fluctuations is adopted. Analytic continuation from $i \Omega_{v}$ to $\omega_{\mathrm{th}}+i \eta$
( $\eta$ being a positive infinitesimal) is eventually required. To compare theoretical results with the experimental spectra, we have to convert from $\omega_{\text {th }}$ to $\omega=\omega_{\mathrm{RF}}-\omega_{\gamma \beta}$, where $\omega_{\gamma \beta}$ is the atomic hyperfine frequency of the transition. Besides being appropriate to the BCS $\left(\left(a_{\alpha \beta} k_{\mathrm{F}}\right)^{-1} \lesssim-1\right)$ and unitarity $\left(\left(a_{\alpha \beta} k_{\mathrm{F}}\right)^{-1} \approx 0\right)$ regimes, the DOS and AL contributions are also able to recover the two-body (molecular) calculation of ref. 25 when the BEC regime $\left(1 \lesssim\left(a_{\alpha \beta} k_{\mathrm{F}}\right)^{-1}\right)$ is approached. (In the text, we have set $a_{\alpha \beta}=a_{\mathrm{i}}$.) Note that the DOS contribution of Fig. $4 \mathrm{a}$ has already been considered in ref. 26 above $T_{\mathrm{c}}$ and in ref. 10 below $T_{\mathrm{c}}$.

Numerical procedures. Owing to the involved structure of the AL contribution (see Fig. 4b), for the external Matsubara frequency $\Omega_{v}$ analytic continuation from the points $i \Omega_{v}$ on the imaginary axis to (just above) the real frequency axis cannot be achieved by the elementary methods that proved sufficien $t^{10}$ for the BCS-RPA approach below $T_{\mathrm{c}}$. The delicate analytic continuation at finite temperature that is required for the AL diagram has been carried out in the literature by resorting to approximations $s^{19}$ that do not apply to the present case, when experimental radiofrequency spectra with non-trivial frequency structures need be accounted for in a variety of situations.

To this end, we have carried out the analytic continuation of the AL contribution resorting to Padé approximants ${ }^{27}$, whereby the dependence on the external frequency $\Omega_{v}$ is approximated by the ratio of two polynomials (the denominator having one extra power with respect to the power $M$ of the numerator). The $2 M$ unknown coefficients have been determined by sampling over about 450 Matsubara frequencies (not necessarily equally spaced). The reliability of the numerical results has been tested against the DOS contribution above $T_{\mathrm{c}}$ as well as the BCS-RPA calculation below $T_{\mathrm{c}}$, which can both be obtained by direct analytic continuation without resorting to Padé approximants. Further independent tests on the numerical procedure are the values of the sum rules for the spectral $\mathrm{area}^{10}$ and its first moment ${ }^{9}$, which are reproduced within $1 \%$ and $10 \%$, in the order.

The energy scale $\Delta_{\infty}$ of the pairing interaction. The most direct way to introduce the quantity $\Delta_{\infty}$ is through the coefficient $\left(3 / 2^{5 / 2}\right) \Delta_{\infty}^{2}$ of the $\omega^{-3 / 2}$ behaviour of the DOS and AL contributions for large $\omega$, which identifies

$$
\Delta_{\infty}^{2}=\int \frac{\mathrm{d} \mathbf{q}}{(2 \pi)^{3}} \frac{1}{\beta} \sum_{\Omega_{v}} \mathrm{e}^{i \Omega_{\nu} \eta} \Gamma_{\alpha \beta}\left(\mathbf{q}, \Omega_{\nu}\right)
$$

where $\mathbf{q}$ is a wave vector and $\Gamma_{\alpha \beta}$ is the pairing propagator of Fig. $4 \mathrm{c}$. This expression is conveniently evaluated by using the spectral representation ${ }^{28}$ of $\Gamma_{\alpha \beta}$. In the BCS regime, $\Delta_{\infty}$ coincides with the absolute value $2 \pi\left|a_{\mathrm{i}}\right| n / m$ of the mean-field shift for temperatures smaller than $\left(m a_{\mathrm{i}}^{2}\right)^{-1}$, whereas in the BEC regime the relation $\Delta_{\infty}^{2}=4 \pi n /\left(m^{2} a_{\mathrm{i}}\right)$ holds in analogy with the BCS mean-field result. Close to $T_{\mathrm{c}}$ at unitarity, the numerical value $0.75 E_{\mathrm{F}}$ of $\Delta_{\infty}$ is comparable to the value $0.8 E_{\mathrm{F}}$ of the pseudogap extracted from the single-particle spectral function ${ }^{11}$. The temperature dependence of $\Delta_{\infty}$ is rather weak for all coupling regimes, extending up to several times the Fermi temperature $T_{\mathrm{F}}$.

The energy scale $\Delta_{\infty}$ is also related to the asymptotic behaviour of the wave-vector distribution function $n(\mathbf{k})$ as obtained from the fermionic single-particle propagator $\beta-\beta$ dressed by the self-energy $\Sigma_{\beta}$ of Fig. $4 \mathrm{c}$. We get $n(\mathbf{k})=\left(m \Delta_{\infty}\right)^{2} / \mathbf{k}^{4}$, where $\Delta_{\infty}^{2}$ is defined as above. From this expression, we identify $\left(m \Delta_{\infty}\right)^{2}$ with the contact intensity $C$ of ref. 8 .

Received 3 October 2008; accepted 8 July 2009; published online 16 August 2009

\section{References}

1. Eagles, D. M. Possible pairing without superconductivity at low carrier concentrations in bulk and thin-film superconducting semiconductors. Phys. Rev. 186, 456-463 (1969).

2. Leggett, A. J. in Proc. 16th Karpacz Winter School Theor. Phys. (eds Pekalski, A. \& Przystawa, J.) 13-27 (Springer, 1980).

3. Noziéres, P. \& Schmitt-Rink, S. Bose condensation in an attractive fermion gas: From weak to strong coupling superconductivity. J. Low Temp. Phys. 59, 195-211 (1985).

4. Ketterle, W. \& Zwierlein, M. W. in Proc. Internat. School Phys. Enrico Fermi, Course 164 (eds Inguscio, M., Ketterle, W. \& Salomon, C.) 95-287 (IOS Press, 2008).

5. Chin, C. et al. Observation of the pairing gap in a strongly interacting Fermi gas. Science 305, 1128-1130 (2004)

6. Shin, Y., Schunck, C. H., Schirotzek, A. \& Ketterle, W. Tomographic rf spectroscopy of a trapped Fermi gas at unitarity. Phys. Rev. Lett. 99, 090403 (2007).

7. Schunck, C. H., Shin, Y., Schirotzek, A. \& Ketterle, W. Determination of the fermion pair size in a resonantly interacting superfluid. Nature 454, 739-743 (2008).

8. Tan, S. Large momentum part of a strongly correlated Fermi gas. Ann. Phys. 323, 2952-2970 (2008). 
9. Yu, Z. \& Baym, G. Spin-correlation functions in ultracold paired atomic-fermion systems: Sum rules, self-consistent approximations, and mean fields. Phys. Rev. A 73, 063601 (2006).

10. Perali, A., Pieri, P. \& Strinati, G. C. Competition between final-state and pairing-gap effects in the radio-frequency spectra of ultracold Fermi atoms. Phys. Rev. Lett. 100, 010402 (2008).

11. Perali, A., Pieri, A., Strinati, G. C. \& Castellani, C. Pseudogap and spectral function from superconducting fluctuations to the bosonic limit. Phys. Rev. B 66, 024510 (2002)

12. Damascelli, A. Probing the electronic structure of complex systems by ARPES. Phys. Scr. T109, 61-74 (2004).

13. Stewart, J. T., Gaebler, J. P. \& Jin, D. S. Using photoemission spectroscopy to probe a strongly interacting Fermi gas. Nature 454, 744-747 (2008).

14. Aslamazov, L. G. \& Larkin, A. I. Effect of fluctuations on the properties of a superconductor above the critical temperature. Sov. Solid State 10, 875-880 (1968).

15. Maki, K. Critical fluctuation of the order parameter in a superconductor. Prog. Theor. Phys. 40, 193-200 (1968)

16. Thompson, R. S. Microwave, flux flow, and fluctuation resistance of dirty type-II superconductors. Phys. Rev. B 1, 327-333 (1970).

17. Skocpol, W. J. \& Tinkham, M. Fluctuations near superconducting phase transitions. Rep. Prog. Phys. 38, 1049-1097 (1975).

18. Abrikosov, A. A. Fundamentals of the Theory of Metals Ch. 19 (North-Holland, 1988)

19. Varlamov, A. \& Larkin, A. Theory of Fluctuations in Superconductors Ch. 7 (Oxford Scholarship Online, 2007).

20. Basu, S. \& Mueller, E. J. Final-state effects in the radio frequency spectrum of strongly interacting fermions. Phys. Rev. Lett. 101, 060405 (2008).

21. He, Y., Chien, C.-C., Chen, Q. \& Levin, K. Temperature and final state effects in radio frequency spectroscopy experiments on atomic Fermi gases. Phys. Rev. Lett. 102, 020402 (2009).
22. Schirotzek, A., Shin, Y., Schunck, C. H. \& Ketterle, W. Determination of the superfluid gap in atomic Fermi gases by quasiparticle spectroscopy. Phys. Rev. Lett. 101, 140403 (2008).

23. Braaten, E. \& Platter, L. Exact relations for a strongly interacting Fermi gas from the operator product expansion. Phys. Rev. Lett. 100, 205301 (2008).

24. Werner, F., Tarruell, L. \& Castin, Y. Number of closed-channel molecules in the BEC-BCS crossover. Eur. Phys. J. B 68, 401-415 (2009).

25. Chin, C. \& Julienne, P. S. Radio-frequency transitions on weakly bound ultracold molecules. Phys. Rev. A 71, 012713 (2005).

26. Massignan, P., Bruun, G. M. \& Stoof, H. T. C. Spin polarons and molecules in strongly interacting atomic Fermi gases. Phys. Rev. A 78, 031602 (2008).

27. Vidberg, H. J. \& Serene, J. W. Solving the Eliashberg equations by means of N-point Padé approximants. J. Low. Temp. Phys. 29, 179-192 (1977).

28. Pieri, P., Pisani, L. \& Strinati, G. C. BCS-BEC crossover at finite temperature in the broken-symmetry phase. Phys. Rev. B 70, 094508 (2004).

29. Bartenstein, M. et al. Precise determination of ${ }^{6} \mathrm{Li}$ cold collision parameters by radio-frequency spectroscopy on weakly bound molecules. Phys. Rev. Lett. 94, 103201 (2005).

30. Simonucci, S., Pieri, P. \& Strinati, G. C. Broad vs. narrow Fano-Feshbach resonances in the BCS-BEC crossover with trapped Fermi atoms. Europhys. Lett. 69, 713-718 (2005)

\section{Acknowledgements}

This work was partially supported by the Italian MUR under contract PRIN-2007 'Ultracold Atoms and Novel Quantum Phases'. This letter is dedicated to the memory of F. Bassani.

\section{Additional information}

Supplementary information accompanies this paper on www.nature.com/naturephysics. Reprints and permissions information is available online at http://npg.nature.com/ reprintsandpermissions. Correspondence and requests for materials should be addressed to P.P. 